\title{
Que répondent les cliniciens enseignants à des demandes pédagogiques de résidents lors de discussions de cas et pourquoi ?
}

\section{What responses do preceptors provide to residents during case discussions about educational requests, and why?}

\section{Luc CÔTÉ ${ }^{1}$ et Georges BORDAGE ${ }^{2}$}

1 Département de médecine familiale et de médecine d'urgence, Faculté de médecine, Université Laval, Québec, Canada

2 Department of Medical Education, College of Medicine, University of Illinois at Chicago, USA

Manuscrit soumis le 22 janvier 2016 ; commentaires éditoriaux formulés aux auteurs le 14 et le 21 mars 2016 ; accepté pour publication le 21 mars 2016

\section{Mots-clés \\ Rétroaction ; stage clinique ; clinicien enseignant ; discussion de cas \\ Keywords Feedback; clerkship; internship; clinical preceptor; case discussion}

\begin{abstract}
Résumé - Contexte et problématique : Les réponses des cliniciens enseignants lorsqu'ils fournissent une rétroaction à un étudiant (externe, interne, résident) en contexte de stage clinique et les cadres conceptuels sur lesquels ils appuient ces réponses restent encore incomplètement documentés. Exégèse : Cette contribution résume les résultats de deux études qui se sont attaché à en faire un inventaire méthodique et systématique, en soulignant l'exploitation pédagogique qui peut en être faite.

Abstract - Background: Responses and conceptual frameworks used by clinical preceptors when giving feedback to students (clerks, interns, residents) during clinical rotations are not well documented. Findings: Inventories of responses and frameworks from two systematic and methodical studies are summarized along with suggestions for instruction and faculty development.
\end{abstract}

\section{Les articles commentés}

Côté L, Bordage. Content and Conceptual Frameworks of Preceptor Feedback Related to Residents' Educational Needs. Acad Med 2012;87:1274-81.

Côté L, Gromaire P, Bordage G. Content and Rationale of Junior and Senior Preceptors Responding to

Residents' Educational Needs Revisited. Teach Learn Med 2015;27:299-306 


\section{Problématique}

Les cliniciens enseignants jouent un rôle essentiel dans le développement des compétences des futurs professionnels en sciences de la santé, en particulier pendant la rétroaction lors de la supervision de cas. D' ailleurs, l'habileté à structurer et à échanger une rétroaction de qualité représente une des compétences clés d'un clinicien enseignant efficace et contribue à l'ouverture des étudiants à l'apprentissage.

\section{Ce que les auteurs ont fait}

En 2012, Côté \& Bordage se sont intéressé au contenu de la rétroaction (ce qui est dit) de 25 cliniciens enseignants en médecine familiale et en médecine interne de l'Université Laval en réponse à des demandes pédagogiques de résidents lors de la discussion de cas ${ }^{[1]}$. Ils ont aussi répertorié les cadres conceptuels (rationnel) guidant les réponses des cliniciens. Un cadre conceptuel est une façon de se représenter comment les choses fonctionnent, et émane selon les cas de théories, de modèles ou de corpus de savoirs élaborés selon la perspective de la pratique basée sur des preuves ${ }^{[2]}$.

Lors d'une rencontre individuelle enregistrée, chaque clinicien a répondu à six courtes vignettes cliniques écrites mettant chacune en évidence une demande pédagogique explicite de la part d'un résident (par exemple, "Que me conseillez-vous de lire ?» ou encore «J'ai peur d'avoir raté quelque chose d'important. »). Dans la première partie de la rencontre, l'enseignant répondait à chaque vignette, selon la méthode du raisonnement à voix haute. Dans la seconde partie, il/elle était invitée à expliquer les bases ou le rationnel de ses réponses. Parmi les résultats, les auteurs ont constaté que : 1) l'utilisation de cadres conceptuels explicites était associée positivement à une plus grande variété de réponses et 2) comparativement aux cliniciens ayant moins d'expérience en supervision, les plus expérimentés utilisaient davantage de cadres conceptuels, donnaient davantage de réponses et posaient davantage de questions stimulantes, posant un défi aux résidents (par exemple, en demandant au résident d'anticiper et d'expliquer le résultat des examens complémentaires prescrits), allant au-delà de la simple clarification (par exemple, en se limitant à demander si tel examen complémentaire a été prescrit).

La même équipe a alors décidé de réaliser une seconde étude sur le même sujet ${ }^{[3]}$, mais en définissant mieux et en comparant les cliniciens enseignants junior et senior ( 20 juniors et 20 seniors), ainsi qu'en diversifiant leur échantillon (trois programmes de résidence et trois facultés de médecine). Les questions de recherche étaient : 1) Est-ce que les cliniciens plus expérimentés donnent plus de réponses et une plus grande variété de réponses que les cliniciens moins expérimentés ?2) Est-ce qu'ils posent plus de questions stimulantes ? et 3) Est-ce qu'ils utilisent plus de cadres conceptuels et une plus grande variété de cadres ? La méthodologie était la même que celle de 2012.

\section{Ce que les auteurs ont trouvé}

La liste des réponses spécifiques de 2012 a alors été enrichie, de 96 à 126 items, et la liste de cadres conceptuels a été doublée, de 16 à 32. Contrairement à 2012, aucune différence n'a été observée entre les juniors et les seniors quant au nombre et à la diversité des réponses aux vignettes. Par contre, lors des réponses, les seniors ont posé davantage de questions de clarification et ont plus souvent fait état de réflexions sur le processus d'apprentissage que les juniors. La spécialité des cliniciens, ainsi que le fait d'avoir ou pas de formation pédagogique n'a pas influencé le nombre et la diversité des réponses, ni des cadres conceptuels.

Un exemple de vignette qui a été soumise aux cliniciens enseignants est fourni dans l'encadré, ainsi que les principales réponses et cadres conceptuels s'y rapportant.

\section{Ce que les auteurs proposent comme retombées pédagogiques}

Ces deux études complémentaires s'appuient sur une démarche scientifique originale et rigoureuse, et leurs résultats peuvent servir à guider, entre autre, la 
production de matériel pédagogique documenté, matériel qui s'inscrit dans la recherche des meilleurs faits probants en éducation médicale. Pour les cliniciens enseignants, les 126 réponses aux six demandes pédagogiques distinctes constituent un riche répertoire où puiser, car ces réponses sont non seulement majoritairement spécifiques à chaque demande pédagogique, mais elles sont diversifiées quant à l'intention ou au but pédagogique visé (par exemple, clarification, conseil, question stimulante, réflexion). L'utilisation de cette liste, en particulier lors d'activités de formation professorale, peut leur être utile, quel que soit leur niveau d'expérience, en les aidant à prendre davantage conscience de leurs réponses, voire à enrichir le répertoire de leurs réponses lors de discussions de cas avec les externes, les internes et les résidents. D'ailleurs, plusieurs cliniciens junior et senior ont constaté son utilité lors de présentations et d'activités de formation pédagogique qui furent réalisées sur ce sujet depuis 2012.

De même, la liste des cadres conceptuels présente aussi un intérêt pour la formation professorale. Elle contribue à aider les cliniciens à identifier les bases conceptuelles (ou le rationnel) de leurs réponses qu'ils ont souvent implicitement en mémoire, à les expliciter, à évaluer si leur compréhension est juste et finalement à associer et discuter les interventions pédagogiques les plus pertinentes découlant des cadres retenus.

Bref, la liste des réponses et des cadres conceptuels découlant de ces deux études s'ajoute aux outils servant à structurer la pratique réflexive des cliniciens enseignants lors de leur rétroaction aux externes et résidents pendant de la discussion de cas.

\section{Encadré. Des réponses aux questions des étudiants et des cadres conceptuels utilisables par les cliniciens enseignants lors d'une rétroaction pédagogique}

Les exemples qui suivent ont été recueillis auprès des cliniciens ayant participé à l'étude analysée ${ }^{[3]}$, en lien avec une vignette pédagogique (en l'occurrence la vignette 4). Les réponses sont catégorisées au regard d'une typologie de la rétroaction du clinicien enseignant ; les cadres conceptuels rendent compte des différentes catégories de références qui inspirent ces réponses.

\section{Vignette 4 : «Auriez-vous des trucs à me donner avec ce genre de patiente non observante?»}

Je voudrais vous parler de Mme Tremblay. Elle a 76 ans et a un diabète, type 2. Je viens de la voir avec sa fille. Je suis le nouveau résident qui s'occupe d'elle, le précédent ayant terminé sa résidence. C'était la Dre Dubé. À chaque fois que je vois Mme Tremblay, elle me parle de cette résidente et me dit combien elle est triste de son départ. C'est clair qu'elle n'a pas fait son deuil. En tout cas, avant aujourd'hui, j'ai vu Mme Tremblay une seule fois et c'était il y a deux mois. Ses glycémies étaient élevées ; j'ai augmenté sa médication et l'ai référée à une diététiste. Aujourd'hui ses glycémies sont encore élevées. J'ai regardé avec elle ce qui s'était passé depuis le dernier rendez-vous pour me rendre compte qu'elle n'avait pas respecté les termes du contrat sur lequel nous nous étions accordés la dernière fois : elle devait surveiller sa diète, surtout les petits biscuits au chocolat et effectuer deux ou trois petites marches par semaine. La dernière fois, $\mathrm{j}$ 'avais pris la peine de bien vérifier si elle était d'accord avec ça. Aujourd'hui elle me dit que pour les marches, elle n'avait pas eu le temps, et que, pour la diète, elle avait eu beaucoup de visites ces derniers temps. En entendant ça, sa fille n'était pas contente du tout. Moi non plus d'ailleurs. J'ai gentiment dit à Madame Tremblay que si elle ne collaborait pas, c'est certain que ses problèmes de santé s'aggraveraient. Je lui ai dit qu'elle pourrait peut-être avoir des problèmes aux reins et peut-être devoir aller en dialyse. Elle s'est mise à pleurer et sa fille m'a dit que je n'étais pas très gentil de faire pleurer 


\section{Encadré. Suite}

sa mère. J'avoue que je ne sais plus trop quoi faire. Auriez-vous des trucs à me donner avec ce genre de patiente-là?

Exemples de réponses utilisables dans le cadre de la rétroaction à l'étudiant concerné par la vignette 4, et leurs buts pédagogiques.

\begin{tabular}{|l|l|}
\hline \multicolumn{1}{|c|}{ Réponse } & $\begin{array}{l}\text { But } \\
\text { pédagogique }\end{array}$ \\
\hline Demander au résident d'expliquer la (les) cause(s) de ses difficultés & Qs \\
\hline Demander au résident s'il a exploré les inquiétudes ou les attentes du patient & $\mathrm{Cl}$ \\
\hline $\begin{array}{l}\text { Demander au résident comment il a conseillé la patiente sur son changement d'habitudes } \\
\text { de vie }\end{array}$ & $\mathrm{Cl}$ \\
\hline Noter la difficulté du résident à établir une alliance thérapeutique & $\mathrm{Re}$ \\
\hline $\begin{array}{l}\text { Explorer comment le résident procède habituellement pour établir une bonne } \\
\text { relation médecin-patient }\end{array}$ & $\mathrm{Cl}$ \\
\hline Souligner l'importance d'explorer l'alliance thérapeutique & $\mathrm{Co}$ \\
\hline $\begin{array}{l}\text { Conseiller sur l'intervention auprès de la personne accompagnant le patient (e.g. explorer } \\
\text { ses inquiétudes) }\end{array}$ & $\mathrm{Co}$ \\
\hline Conseiller sur les manières de se centrer sur le patient (et ses proches) & $\mathrm{Co}$ \\
\hline Conseiller sur les étapes de changement d'habitudes de vie & Co \\
\hline $\begin{array}{l}\text { Mettre en garde au sujet des conséquences négatives de ne pas impliquer le patient dans le } \\
\text { processus de changement d'habitudes de vie }\end{array}$ & Co \\
\hline Cl : Demande de clarification ; Qs : Question stimulante ; Re : Réflexion du clinicien enseignant ; Co : Conseil \\
\hline
\end{tabular}

Exemples de cadres conceptuels inspirant la rétroaction à l'étudiant concerné par la vignette 4 , et leur nature**.

\begin{tabular}{|l|l|}
\hline Cadre conceptuel & Nature \\
\hline L'alliance thérapeutique & Reco \\
\hline $\begin{array}{l}\text { Les étapes progressives du changement d'habitudes } \\
\text { de vie }\end{array}$ & Mo \\
\hline L'approche centrée sur le patient & Mo \\
\hline Transfert - contre-transfert & Mo \\
\hline Double agenda d'entrevue : patient et médecin & Mo \\
\hline Rétroaction constructive & Mo \\
\hline $\begin{array}{l}\text { L'approche centrée sur l'étudiant (prendre ses } \\
\text { besoins en compte) }\end{array}$ & Mo \\
\hline$* *$ Reco : Recommandation de bonne pratique ; Mo : Modèle ou approche \\
\hline
\end{tabular}




\section{Références}

1. Côté L, Bordage. Content and Conceptual Frameworks of Preceptor Feedback Related to Residents'Educational Needs. Acad Med 2012;87:1274-81.

2. Bordage G. Conceptual Frameworks to Illuminate and Magnify. Med Educ 2009;43:312-9.
3. Côté L, Gromaire P, Bordage G. Content and Rationale of Junior and Senior Preceptors Responding to Residents' Educational Needs Revisited. Teach Learn Med 2015;27:299-306.

Correspondance et offprints : Luc Côté. Département de médecine familiale et de médecine d'urgence, Faculté de médecine, Université Laval, G1V 0A6 Québec, Canada.

Mailto : luc.cote@fmed.ulaval.ca 